\title{
Trivium
}

Revue franco-allemande de sciences humaines et sociales - Deutsch-französische Zeitschrift für Geistesund Sozialwissenschaften

15 | 2013

La science pense en plusieurs langues

\section{Tributaire de sa propre langue}

\section{Jürgen Trabant}

Traducteur : Claudine Layre et Anne-Emmanuelle Fournier

\section{(2) OpenEdition}

\section{Journals}

Édition électronique

URL : http://journals.openedition.org/trivium/4701

ISSN : 1963-1820

\section{Éditeur}

Les éditions de la Maison des sciences de l'Homme

\section{Référence électronique}

Jürgen Trabant, «Tributaire de sa propre langue », Trivium [En ligne], 15 | 2013, mis en ligne le 09 décembre 2013, consulté le 08 septembre 2020. URL : http://journals.openedition.org/trivium/4701

Ce document a été généré automatiquement le 8 septembre 2020

\section{(c) (i) (9)}

Les contenus des la revue Trivium sont mis à disposition selon les termes de la Licence Creative Commons Attribution - Pas d'Utilisation Commerciale - Pas de Modification 4.0 International. 


\title{
Tributaire de sa propre langue
}

\author{
Jürgen Trabant \\ Traduction : Claudine Layre et Anne-Emmanuelle Fournier
}

Quiconque s'occupe de philosophie et d'art appartient plus particulièrement à sa patrie que tout autre, j'en ai encore fait l'expérience ici sur mon frère Alexander et moi-même. J'ai aimé être à Paris au moins autant que lui, peut-être même plus encore, cependant lui se sentait infiniment moins étranger ici. Les échanges dans l'un et l'autre sens n'ont jamais fait problème pour lui. Pour la philosophie et l'art nous dépendons davantage de notre propre langue, que la sensibilité et la disposition d'esprit ont forgée et par laquelle elles sont formées en retour. (Wilhelm von Humboldt à Goethe le 18 mars 1799)

1 Je me suis déjà exprimé plusieurs fois publiquement sur le thème de la mondialisation et de la « langue du pays ", comme le formule avec tant de diplomatie le programme de notre colloque. (Une précision pour nos amis français : en Allemagne, on n'a en effet pas le droit d'utiliser l'adjectif national, employé dans la version française du programme, sans que ne soit agité le spectre du nationalisme voire du nazisme. D'où le choix politiquement neutre de l'expression « langue du pays » au lieu du vilain mot en n-. Je me suis surtout demandé pourquoi les Allemands - contrairement aux Français sont si empressés d'abandonner leur langue nationale, pardon, la langue de leur pays. Je présume que nos invités français, après avoir pris un shuttle pour quitter l'airport, suite à l'achat d'un bus ticket à l'information center, sont passés devant les job centers où la task force de la ministre de l'Éducation était in action (brain up !), puis ont regardé un peu de TV publicity dans leur hotel lounge et se demandent de toute façon de quel national language il pourrait bien être question en Germany. What are we talking about? Come in and find out.

2 Mais aujourd'hui, je suis très obéissant et je vais donc supposer que nous parlons de la vieille langue nationale, autrement dit de ce qu'on appelle l'allemand, das Deutsche (voilà encore un vilain mot dans ce pays, the $d$-word). Je me concentre donc sur la question posée par les organisateurs : «Enseigner et publier dans la langue nationale : nécessité fondamentale ou reliquat?»

3 Il s'agit évidemment de savoir si la langue nationale est le fondement de la science, et si tel est le cas, si elle doit le rester, ou si elle ne l'est pas mais n'est plus que le reliquat 
de temps révolus dans le meilleur des mondes de l'actuelle "globanglicisation»? Habituellement, j'aborde cette question d'un point de vue historique, mais cela demande toujours beaucoup de temps. Comme je n'en ai pas, je serai là aussi obéissant et l'analyserai pour une fois sous un angle actuel et systématique. Je parlerai d'abord de l'acte de publier, puis de l'enseignement et enfin de nouveau de l'écriture.

\section{Publier}

4 En Allemagne, les sciences naturelles publient en «globalais ». L'un de nos grands spécialistes en sciences naturelles l'avait déjà constaté et revendiqué il y a vingt ans : «La recherche de pointe - il pensait bien sûr aux sciences dures - parle anglais.» Cependant, les autres sciences, les sciences sociales et surtout les sciences de la culture publient tout de même la plupart du temps dans la vieille langue nationale.

5 Mais cela ne fait rien, diraient les politiques allemands chargés de la culture et des sciences qui veulent remettre de l'ordre dans ce pays. Cela ne fait rien parce qu'on ne peut de toute façon pas remporter de prix Nobel et encore moins gagner de l'argent grâce aux sciences de la culture. En tout cas, pour l'instant, cela ne fait rien. Mais cela doit évidemment changer à terme : c'est une vieillerie, un résidu, un reliquat. Les hommes politiques de ce pays ont déjà répondu depuis longtemps à notre question. Nous nous accordons cependant encore une fois le droit de demander : la situation doitelle vraiment changer, ou cette vieillerie est-elle peut-être le fondement même de ces sciences?

6 La constatation selon laquelle les sciences naturelles se font en globalais tandis que les autres sciences s'élaborent dans la langue nationale est suffisamment attestée scientifiquement (Ammon). Je l'ai cependant vérifiée encore une fois à l'aide de la liste des publications des membres de l'Académie de Berlin (annuaire 2000), dans les bâtiments de laquelle nous nous trouvons. L'observation d'Ammon est exacte : il n'y a plus que très peu de publications de sciences naturelles en allemand; quelques-unes en médecine, un manuel allemand de chimie et quelques articles de vulgarisation d'un neurobiologiste. Dans ce domaine, il est donc manifeste que la langue nationale ne sert plus qu'à apporter la bonne parole aux autochtones. Toutefois, il s'agit déjà en soi d'une dimension très importante - j'y reviendrai tout de suite.

7 Les techniciens écrivent pour le moment davantage en allemand qu'en globalais. Cela ne nous étonnera guère puisque ce sont précisément eux, ou encore les savants œuvrant dans des domaines scientifiques proches de l'artisanat, qui à partir du $\mathrm{XV}^{\mathrm{e}}$ / $\mathrm{XVI}^{\mathrm{e}}$ siècle ont introduit l'usage des « langues nationales » en Europe, à la place du latin qui était le globalais de l'époque. Quant à la psychologie, elle s'écrit en allemand ou en anglais selon son orientation. Dans les sciences humaines et sociales, les linguistes, les politologues, les sociologues écrivent parfois en anglais mais continuent à utiliser l'allemand. En dépit de ce domaine intermédiaire - plutôt réduit - on constate une nette division linguistique entre les deux champs scientifiques. Dans les sciences naturelles, on écrit en globalais, tandis que les langues nationales dominent dans les sciences humaines (et sociales). Qui oserait nier qu'il y ait deux cultures. La division n'a jamais été aussi nette qu'aujourd'hui. Et ce que Snow $^{1}$ n'avait pas du tout pris en compte, c'est que ce clivage correspond aussi à deux mondes linguistiques différents. 


\section{Enseigner}

8 Dans le contexte de l'enseignement, la situation est un peu différente : la division entre ces deux cultures n'est pas encore consommée. En parcourant la liste des cours proposés dans les deux universités berlinoises, j'ai été surpris de constater qu'on enseigne encore relativement peu en anglais. Les cours de sciences naturelles commencent à peine à être donnés dans cette langue. C'est le cas seulement dans certains domaines de recherche «de pointe» très précis, par exemple en neurobiologie, en microchimie ou en informatique, mais même dans ces matières, cela n'arrive quasiment jamais lors des premières années de faculté. Dans certains masters ou séminaires de recherche spécialisés, on parle en revanche le globalais.

9 Les sciences sociales sont elles aussi très peu enseignées en anglais. On opte pour l'anglais quand il s'agit de thèmes spécifiquement américains ou quand le chargé de cours ne sait pas l'allemand. Pour parler des relations internationales ou des processus économiques internationaux, on parle l'anglais globalisé, ce qui est cohérent. En revanche, je m'attendais à du management anglophone dans les cours de gestion, puisqu'en Germany, chez Siemens à Munich et dans toutes les grandes entreprises, on parle anglais, mais cela ne se reflète pas dans les programmes universitaires.

10 Les sciences de la culture - sauf les études de langues et littératures anglo-américaines bien sûr - sont enseignées dans la langue nationale.

11 Mais bien entendu, le conseil d'administration de mon université a déjà décidé depuis longtemps qu'il fallait mettre sans tarder un terme à cette monstruosité et que tous les cours devraient le plus tôt possible avoir lieu en globalais. Nous avons évidemment aussi un commissaire linguistique qui a l'oreille du président de l'université, obsédé par l'innovation, et qui veut de toutes ses forces promouvoir le globalais dans l'enseignement universitaire. Et tous trouvent ça très bien, plutôt moderne, et estiment qu'il doit en être ainsi. Et maintenant, on nous rabat les oreilles avec l'“Université d'élite". L'élite, de toute façon, parle l'anglais. Alors qu'on s'y mette, et vite!

12 La réalité de l'enseignement universitaire est encore très éloignée du meilleur des anglo-mondes qui a été fixé comme objectif. Et je n'ai pas envie de savoir à quoi il ressemble là où il existe déjà, autrement dit de savoir à quel point le cours donné en anglais par mon collègue chimiste est stimulant, spirituel, nuancé. Le cours en anglais ne peut être bon que si M. le professeur a vécu des années en Angleterre ou aux ÉtatsUnis et s'il a enseigné là-bas, sinon il est impossible de parvenir à un bon cours en globalais. Tout professeur de langue le sait.

13 Toutefois, nous rencontrons ici un petit problème que nos innovateurs n'ont jamais vraiment pris en compte. Il faut d'abord exporter les scientifiques pour les rendre capables de bien enseigner en anglais ici en Allemagne. (Le danger qu'ils disparaissent à cette occasion est bien sûr assez élevé : quasiment personne ne revient des États-Unis, en tout cas aucun neurobiologiste ou informaticien qui a vécu en Terre promise avec des émoluments somptueux, des conditions de recherche fantastiques, des étudiants motivés parce que payant leurs études, et très peu d'heures de cours - entre deux et quatre, par comparaison aux huit heures ici). On ne peut raisonnablement espérer que grâce aux cours donnés en anglais par leur professeur de chimie nos étudiants apprennent si bien cette langue qu'ils puissent ensuite eux-mêmes l'employer brillamment et de façon nuancée dans leur futur enseignement. 
14 Prenons un exemple. Comme je ne peux pas espionner mon collègue de chimie, prenons-en un autre, moins compromettant : le mien. Je pense être capable de faire des cours tout à fait corrects en français ou en italien, je le fais d'ailleurs parfois, fort de mon bagage dans les langues romanes (et cela me plaît). Mais j'ai vécu des années en Italie et longtemps en France. Je pourrais aussi donner des cours à peu près acceptables en anglais parce que j'ai enseigné presque deux ans aux États-Unis. Il n'empêche : ma prestation quand j'enseigne dans ces langues n'est tout simplement pas comparable avec ce que je fais quand je parle l'allemand (dont vous me direz qu'il est déjà assez mauvais). Et par conséquent, mon enseignement n'est donc absolument du même niveau que celui de mes homologues de langue maternelle anglaise, française ou italienne dans leurs cours. Nous nous en rendons bien compte à chaque fois que nous leur sommes directement confrontés. C'est tout simplement un avantage considérable que d'avoir le droit d'enseigner (de faire une conférence, de débattre) dans sa langue ou dans la langue qu'on possède le mieux. On est meilleur, un point c'est tout. En tant qu'enseignant, on n'appartient véritablement à l'élite que lorsqu'on dispose de toute la palette des capacités d'expression.

15 Aussi dans la réalité universitaire, dans l'enseignement, dans la pratique orale de la langue, peu de scientifiques sont enthousiastes à l'idée de renoncer à leur langue nationale. Même ceux qui publient en anglais n'enseignent pas forcément dans cette langue, en tout cas pas de leur propre gré. Pourquoi cet état de fait est-il non seulement compréhensible mais en outre adéquat pour la science?

16 Nous, linguistes, distinguons au moins deux dimensions du langage, la sémantique et la pragmatique: la relation au référent et à la réalité d'une part, et la relation à l'interlocuteur, la composante communicative d'autre part. Parler, ce n'est pas seulement désigner des choses mais aussi et en même temps instaurer une relation sociale. Nous ne parlons pas seulement de quelque chose mais aussi toujours avec quelqu'un. Dans le langage de la science, c'est la dimension sémantique qui est première : il y est question des choses, de la juste compréhension du monde.

17 Or, la distinction entre le parler et l'écrit, entre l'enseignement et la publication, fait ressortir la place différente de chacune de ces deux dimensions langagières dans ces domaines respectifs. Entre le travail chez soi et l'amphithéâtre, il y a une dissemblance décisive : l'enseignement, autrement dit la science orale, se fait dans la présence corporelle d'autres personnes. Enseigner, c'est parler de sujets nouveaux dont la compréhension est ardue : la relation sémantique, en d'autres termes la relation à la réalité est difficile. Dans ce cas, il est bon de ne pas s'encombrer en même temps de difficultés dans la dimension relationnelle mais d'utiliser les meilleures possibilités existantes du parler ensemble. Et c'est ce que nous offre - pour le moment encore, en tout cas - la langue nationale. Il est donc cohérent d'utiliser les possibilités communicatives de la langue en usage dans l'environnement immédiat des protagonistes de l'échange. La dimension de la communication est ainsi assurée, de sorte que nous pouvons aborder les sujets ardus sans détour.

18 (C'est d'ailleurs précisément ce qui a été l'acte de libération génial dans l'histoire des sciences, et qui a constitué, comme nous l'avons évoqué, une raison décisive du succès des sciences naturelles modernes, proches des artisanats : s'occuper d'abord des choses et utiliser la langue en usage dans l'environnement naturel de communication pour désigner les choses étudiées, au lieu de commencer par potasser le latin globalisé avant de se consacrer ensuite aux choses, à supposer qu'il reste du temps pour le faire.) 


\section{Écrire}

Il en va différemment de l'écrit: d'abord, la communauté de communication n'est pas la même, et de surcroît, une nette différence apparaît quant aux choses sur lesquelles on écrit.

Premièrement, écrire me libère de la présence de mes interlocuteurs. Les autres ne sont pas là. L'écriture permet donc au scientifique de se concentrer entièrement sur l'objet, sur la dimension sémantique. Il est ici, pour ainsi dire, tout à son affaire et donc tout à la science. Par ailleurs, écrire ouvre par principe un autre espace communicatif que la langue parlée, l'espace de la distance communicative.

1 Étant donné l'ambition universelle de vérité intrinsèque à la science, cet espace est en théorie aussi vaste que l'humanité elle-même. En effet, tout chercheur en sciences naturelles aspire non seulement à être compris par ses étudiants dans l'amphithéâtre mais souhaite que tous les scientifiques du globe prennent connaissance de ses idées. Pour écrire, il utilise donc si possible la langue qui comble le mieux cette distance communicative. Et dans cette optique, en principe, c'est précisément le globalais qui est le plus adapté.

Cependant, toute activité scientifique n'exige pas cet espace globalisé. Il serait ainsi absurde de décrire en globalais des problèmes de droit concernant la législation nationale. La plus grande distance communicative nécessaire ici, c'est la nation, et non l'humanité. La langue nationale suffit. Mais bien sûr, ce n'est pas ainsi qu'on obtient un prix Nobel.

3 Deuxièmement, l'écriture fait apparaître une dissemblance quant aux choses sur lesquelles on écrit, qui constitue sans doute le fondement de la dichotomie constatée au départ entre les deux cultures linguistiques: le monde, les choses sur lesquelles les sciences écrivent ne sont pas semblables. Schématiquement, les objets des sciences naturelles (ou plus généralement des sciences expérimentales) sont les choses, tandis les objets des sciences de la culture_(ou plus généralement des sciences théoriques) sont les textes et les signes produits par les êtres humains. Cela entraîne une disparité essentielle dans l'activité scientifique.

Nous la décrirons ainsi, de façon un peu caricaturale: le spécialiste en sciences naturelles fait quelque chose avec les objets eux-mêmes, que ce soient des insectes, des parties du corps humain, des cristaux, des étoiles, des chromosomes, etc. Il fait quelque chose, il mesure, il pèse, puis dresse un procès-verbal de ses expériences. Ici, l'activité scientifique en elle-même n'est pas le discours mais le «faire-quelque-chose-avec-lesobjets». La langue sert uniquement à la désignation de l'objet étudié (et à la communication), elle vient s'ajouter en quelque sorte post festum, souvent d'ailleurs le scientifique n'a même pas besoin de la langue, un diagramme peut suffire. Les mots ne sont ici que des signes (matériels). On se passe en quelque sorte de leur dimension de sens propre à chaque langue (ce que Saussure appelle le signifié). On fait disparaître ici la langue individuelle en tant que telle, considérée dans sa spécificité. Peu importe au fond dans quelle langue sont désignés les objets étudiés scientifiquement. Au demeurant, le scientifique n'a pas besoin de la langue dans toute sa richesse lexicale et stylistique, mais uniquement dans un registre limité. 
25 Dans les sciences humaines et sociales, il est question du monde de l'être humain qui se manifeste dans les textes et dans les signes. Il s'agit de fixer et de continuer à écrire ces textes et ces signes à travers des commentaires et des interprétations, de conserver ces textes et ces signes dans la langue. Écrire sur les textes et les signes constitue donc l'activité scientifique elle-même. Écrire ne sert pas ici à fixer post festum le résultat d'une recherche effectuée avec de quelconques appareils, donc sans langue ; l'écriture constitue la recherche elle-même. L'écriture scientifique n'est pas ici uniquement une désignation d'objets que j'ai manipulés mais la production d'un texte qui est en tant que tel le résultat de la recherche. De même que le spécialiste de sciences naturelles a besoin des meilleurs appareils (microscope, accélérateur de particules, etc.), le chercheur en sciences humaines et sociales a besoin de son meilleur instrument, à savoir la langue qu'il maîtrise le mieux. Le produit classique de cette activité scientifique est d'ailleurs le livre, un objet linguistique, ce qui représente une différence fondamentale avec les sciences naturelles, dans lesquelles on n'écrit pas de livres à proprement parler. Le livre que je produis devient à son tour une partie de la culture, une partie des textes et des signes. En écrivant, je continue à construire le monde culturel sur lequel j'écris. Et j'aspire à le faire non seulement avec le plus de nuances possible mais également dans une langue qui soit aussi belle, aussi amusante et aussi spirituelle que possible. Les qualités rhétoriques et poétiques de mon texte ne sont pas sans importance. De même que les poètes écrivent dans la langue qu'ils savent le mieux, le chercheur en sciences humaines et sociales doit lui aussi écrire dans la langue qu'il possède le mieux.

26 En général, il s'agit de la langue nationale, la langue dite du pays, dans laquelle il a été élevé. (Mais il n'en est pas forcément ainsi : jusqu'au cours du XVIII ${ }^{\mathrm{e}}$ siècle, pour les "spécialistes en sciences de la culture ", c'était le latin global, c'est ensuite seulement que les différentes langues nationales européennes ont pris cette place).

27 Depuis le XVIII ${ }^{e}$ siècle, les meilleurs ouvrages en sciences de la culture de mon pays ont été rédigés dans la langue nationale. Je ne connais aucun livre éminent de ce domaine qui aurait été écrit en globalais au cours des deux derniers siècles par un scientifique germanophone travaillant en Allemagne (ceci est bien sûr important). Le dernier en date fut peut-être les Prolégomènes à Homère d'August Friedrich Wolf, rédigés en globalais latin en 1795. Il n'existe pas encore de livre équivalent écrit en anglais globalisé par un Allemand. N’en déplaise à Hubert $\mathrm{Markl}^{2}$, dans nos spécialités, la recherche de pointe parle nécessairement la « langue nationale».

28 Toutefois, en raison du lien de mon activité scientifique à la langue que je maîtrise le mieux et qui n'est pas la langue de l'humanité, je suis bien sûr confronté au problème de communication qui nous réunit ici. J'ai besoin de ma langue pour penser, pour élaborer ma pensée et mes écrits scientifiques. Comme le disait Humboldt, je suis tributaire de ma propre langue. C'est la langue qui me permet de déployer la totalité de mes capacités intellectuelles. Seulement, il existe en Europe plus d'une langue de ce type. L'Europe pense en plusieurs langues. C'est d'ailleurs formidable, mais malheureusement, plus personne ne les comprend au-delà des frontières de sa communauté linguistique respective. Mon voisin français ne comprend déjà plus ma langue, sans parler du reste de l'humanité.

29 Et pourtant, chercheur en sciences de la culture, j'ai en vue par principe tout autant l'ensemble de la communauté de communication humaine que le spécialiste de sciences naturelles. Tous ceux qui dans le monde travaillent sur les inscriptions assyriennes 
doivent savoir ce que j'ai à dire. Tous ceux qui veulent comprendre Dante, Racine ou Goethe, doivent être informés de la façon dont je les comprends. Mais ce que j'ai à dire, je ne peux l'exprimer vraiment bien que dans ma propre langue. Et elle n'est pas universelle. Tel est le dilemme, pour nous spécialistes européens en sciences de la culture.

30 Que faire alors, nous autres Européens? Je peux émigrer aux États-Unis et y devenir professeur, l'opposition langue nationale / langue universelle disparaîtra peu à peu d'elle-même. Je peux épouser une Américaine ou un Américain et m'adonner toute ma vie à un bain linguistique (je ne sais pas si c'est conseillé). Comme le souhaitent le président de mon université et son commissaire à la globalisation, je peux commencer à enseigner en anglais dans l'espoir que cela améliorera aussi mes compétences écrites dans cette langue. Mais je le sais : ce ne sera tout simplement pas aussi bon qu'en allemand. Adieu l'élite!

31 L'issue à ce dilemme est bien sûr la traduction. En conclusion, je dirais simplement ceci : des développements précédents ressort la nécessité absolue d'une politique de la traduction scientifique, d'une aide généreuse à l'exportation de nos recherches par le biais d'un programme national de traduction. Cela rendrait nos recherches concurrentielles au niveau international, comme c'est le cas dans les sciences naturelles grâce notamment à la mise à disposition de tous ces beaux appareils onéreux. Le volume financier d'un seul projet en physique (comme le DESY ${ }^{3}$ ) serait satisfaisant, mais celui d'un Institut Max Planck ${ }^{4}$ de taille modeste suffirait déjà amplement.

32 Cependant, une telle globalisation communicative peu chère de nos produits en sciences de la culture n'est pas prévue dans ce pays. La politique culturelle de l'Allemagne emprunte une autre voie, la jacobine. Au lieu de financer le projet girondin de traduction, le gouvernement, l'industrie et la publicité entreprennent le projet jacobin et globalisant beaucoup plus coûteux de reconversion linguistique de l'ensemble du pays. La République française a démontré avec succès comment on peut, sur le plan linguistique, mettre tout un pays au pas, sur la voie irradiante des Lumières. Pourquoi cela ne marcherait-il pas en Allemagne? Enfin, quand il n'y aura plus de langue nationale, nous n'aurons plus besoin de traduire. Et ce serait bien ainsi, diraient ceux qui sont à la tête de ce pays.

33 On this happy note, qui nous ramène au début de mon intervention, et dans laquelle j'ai quand même fini par me montrer insolent, je vous remercie pour votre attention.

\section{NOTES}

1. Snow, Charles Percy (1905-1980) : scientifique et écrivain anglais, auteur de Les deux cultures, J. J. Pauvert, 1968 [N.d.T.].

2. Hubert Markl, né en 1938, biologiste allemand, président de la Société Max Planck de 1996 à 2002, est l'auteur de la citation mentionnée plus haut: «la recherche de pointe parle anglais » [N.d.T.]. 
3. DESY : le «Deutsches Elektronen-Synchrotron " (synchrotron allemand à électrons) est un centre de recherche européen en physique des particules [N.d.T.].

4. Institut Max Planck: la Société Max Planck, équivalent du CNRS français, est constituée de nombreux Instituts du même nom [N.d.T.].

INDEX

Mots-clés : Langue nationale / globalisation / anglais / spécialistes en sciences naturelles et en sciences de la culture / enseigner / publier

Schlüsselwörter : Landessprache / Globalisierung / Englisch / Kultur-und Naturwissenschaftler / lehren / publizieren

\section{AUTEURS}

\section{JÜRGEN TRABANT}

Jürgen Trabant est professeur émérite de linguistique à l'Institut de philologie romane à

l'Université libre de Berlin. Pour en savoir plus, consulter la notice. 